\title{
Investigation of the Current Situation of Medical Education in Japan Based on the Results of Accreditation Based on Global Standards
}

\author{
Yoshikazu Asada*
}

\begin{abstract}
The Japan Accreditation Council for Medical Education (JACME) is conducting an evaluation of medical education programs based on the Global Standards in Japan. A total of 34 institutions have been evaluated by JACME. However, only the individual reports for each institution are available, without any summarized data. This study investigates the current situation of Japanese medical education programs, including their advantages and disadvantages. After summarizing the reports from the 34 institutions, it was found that most reports showed that Area 3 (Assessment of Students) and Area 7 (Program Evaluation) were the weakest points, with many institutions only partly meeting the standards for these areas. The same applies to certain sub-areas of Area 2 (Educational Programme) and Area 6 (Educational Resources). One reason for the low scores of Area 3 and Area 7 is the lack of assessment policies using Information and Communication Technology and the excessive expectations of Institutional Research, including learning analytics. In contrast, almost all institutions met the standards set forth in Area 8 (Governance and Administration). This study has some limitations in that it is based on the results of the JACME reports, which only describe the results of the sub-areas and not those of each standard. More detailed information is needed to further investigate the aspects of Japanese medical education.
\end{abstract}

Keywords: Accreditation, Global Standards, Institutional Research, Medical Education

\section{Introduction}

The evaluation of education programs is one of the most important topics for quality assurance in medical education. This also applies to Japanese medical education. The Japan Accreditation Council for Medical Education (JACME), which was established in 2015, conducts the evaluation and accreditation of medical education programs in Japan. They perform their evaluation based on the Global Standards for Quality Improvement: Basic Medical Education by the World Federation for Medical Education (hereinafter referred to as WFME BME Standards) [1].

\footnotetext{
* Center for Information, Jichi Medical University, Tochigi, Japan
} 
There are various versions of the WFME BME Standards. The 2012 version was revised in 2015. Following this revision, the Japanese version was also updated from version 1.3, which was based on the 2012 WFME BME Standards, to version 2.1, which was based on the 2015 WFME BME Standards. The newest Japanese-translated version is version 2.3, which is also based on the 2015 WFME BME standards and was made only just for a minor translation revision.

The 2015 WFME BME Standards is divided into nine areas and the areas are divided into 35 sub-areas. Table 1 shows the titles of each sub-area and the numbers of standard statements of the 2015 edition.

Table 1: The Names and numbers of areas, sub-areas, and standard statements of the 2015 WFME BME Standards

\begin{tabular}{|c|c|c|}
\hline Areas and Sub-Areas & Basic & Quality \\
\hline \multicolumn{3}{|l|}{ Area 1 Mission and Objectives } \\
\hline 1.1 Mission & 8 & 2 \\
\hline 1.2 Institutional Autonomy and Academic Freedom & 2 & 2 \\
\hline 1.3 Educational Outcomes & 8 & 3 \\
\hline 1.4 Participation in Formulation of Mission and Outcomes & 1 & 1 \\
\hline \multicolumn{3}{|l|}{ Area 2 Educational Programme } \\
\hline 2.1 Framework of the Program & 3 & 1 \\
\hline 2.2 Scientific Method & 3 & 1 \\
\hline 2.3 Basic Biomedical Sciences & 2 & 2 \\
\hline $\begin{array}{l}\text { 2.4 Behavioural and Social Sciences, Medical Ethics, and } \\
\text { Jurisprudence }\end{array}$ & 4 & 3 \\
\hline 2.5 Clinical Sciences and Skills & 5 & 4 \\
\hline 2.6 Program Structure, Composition, and Duration & 1 & 4 \\
\hline 2.7 Program Management & 2 & 2 \\
\hline 2.8 Linkage with Medical Practice and the Health Sector & 1 & 2 \\
\hline \multicolumn{3}{|l|}{ Area 3 Assessment of Students } \\
\hline 3.1 Assessment Methods & 6 & 3 \\
\hline 3.2 Relation between Assessment and Learning & 4 & 2 \\
\hline Area 4 Students & & \\
\hline
\end{tabular}




\begin{tabular}{|c|c|c|}
\hline Areas and Sub-Areas & Basic & Quality \\
\hline 4.1 Admission Policy and Selection & 3 & 3 \\
\hline 4.2 Student Intake & 1 & 1 \\
\hline 4.3 Student Counseling and Support & 4 & 2 \\
\hline 4.4 Student Representation & 5 & 1 \\
\hline Area 5 Academic Staff/Faculty & & \\
\hline 5.1 Recruitment and Selection Policy & 3 & 2 \\
\hline 5.2 Staff Activity and Staff Development & 5 & 2 \\
\hline \multicolumn{3}{|l|}{ Area 6 Educational Resources } \\
\hline 6.1 Physical Facilities & 2 & 1 \\
\hline 6.2 Clinical Training Resources & 3 & 1 \\
\hline 6.3 Information Technology & 2 & 5 \\
\hline 6.4 Medical Research and Scholarship & 3 & 2 \\
\hline 6.5 Educational Expertise & 3 & 3 \\
\hline 6.6 Educational Exchanges & 2 & 2 \\
\hline \multicolumn{3}{|l|}{ Area 7 Programme Evaluation } \\
\hline 7.1 Mechanisms for Program Monitoring and Evaluation & 5 & 4 \\
\hline 7.2 Teacher and Student Feedback & 1 & 1 \\
\hline 7.3 Performance of Students and Graduates & 3 & 5 \\
\hline 7.4 Involvement of Stakeholders & 1 & 3 \\
\hline \multicolumn{3}{|l|}{ Area 8 Governance and Administration } \\
\hline 8.1 Governance & 1 & 3 \\
\hline 8.2 Academic Leadership & 1 & 1 \\
\hline 8.3 Educational Budget and Resource Allocation & 2 & 2 \\
\hline 8.4 Administration and Management & 2 & 1 \\
\hline 8.5 Interaction with Health Sector & 1 & 1 \\
\hline Area 9 Continuous Renewal & & \\
\hline
\end{tabular}




\begin{tabular}{|c|c|c|}
\hline Areas and Sub-Areas & Basic & Quality \\
\hline (no sub-areas) & 3 & 12 \\
\hline
\end{tabular}

There are two standards for each area: Basic Standards and Standards for Quality Improvement. Basic Standards (hereinafter, referred to as "Domain B") must be fulfilled for accreditation, and Standards for Quality Improvement (hereinafter, referred to as "Domain Q") should be fulfilled for accreditation.

Table 1 also shows the number of Domains B and Q in each area and sub-area. The 2015 WFME BME Standards has 106 Domain B, 90 Domain Q, and 127 Annotations. Each standard is evaluated according to three levels: "fulfilled," "partly fulfilled," and "not fulfilled."

Each area also has an Annotations section for clarifying the expressions present in their respective standards. The Japanese-translated edition [2] also has additional sections called "Japanese Annotations," which are based on specific situations pertaining to Japanese medical education, such as the educational program based on the Model Core Curriculum for Medical Education in Japan [3].

There are some previous studies about the accreditation based on the WFME BME Standards. For example, Wajid et al. explored the appropriateness of the Standards to Pakistani context [4]. They conducted a survey of faculty, graduates, and students with a scale to rate the appropriateness. Some standards statements, such as in Area 1 and Area 4, got negative responses because of the different contexts, even though they used "global" standards in their study. Ho et al. conducted case studies of the "Glocalization" of medical school accreditations in Asian countries [5]. They concluded the importance of not only the accreditation practice being in line with the global standards, but also ensuring the local cultural values and societal needs.

Since 2015, the JACME has been evaluating several medical education programs in Japan. However, although the results of each accreditation have been reported on the website, there is no summarized data for them. Summarizing the results of the evaluation could have several benefits: (1) a clarification of the current situation including the advantages and disadvantages of the Japanese medical education system and (2) the provision of suggestions for the improvement of medical education in Japan toward global standards accreditation. Thus, this study aims to analyze the results of these evaluations to clarify the current situation of medical education programs in Japan.

\section{Method}

\subsection{Target}

While there are 82 medical universities and colleges in Japan, only 34 (41\%) have completed accreditation from JACME. Thus, this study analyzed the results of 34 evaluations by the JACME. The results and comments of the accreditations are published on the JACME website (in Japanese) [6]. 


\subsection{Grouping of institutions}

Japanese version 1.3 is based on a different version of the WFME BME Standards and does not have the same number of sub-areas. On the other hand, Japanese versions 2.1, 2.11, 2.2, and 2.3 are all based on the 2015 WFME BME Standards. Thus, the summarized data of accreditations based on version 1.3 (hereinafter, referred to as "Group 1") were used independently from those done after version 2.1 (hereinafter, referred to as "Group 2").

\subsection{Analyzing of reports}

Since the reports do not include the results based on each standard statement, the author counted the results of the sub-areas. Since Group 1 uses old versions of the standards, the numbers of standards are different from Group 2. In addition, some of the statements of standards are a little outdated. Therefore, the reports of Group 1 were used only for (1) comparing the fulfilled ratio between Domain B and Domain Q and (2) the discussion with the accreditation comments by JACME.

\subsection{Statistical test}

The results of accreditation judgments are announced based on the sub-areas. As an example, area 1 has four sub-areas, so four different judgments are announced to the institutions; this could take the form of 1.1 and 1.2 are fulfilled and 1.3 and 1.4 are partly fulfilled. Based on this, a chi-squared test was used to determine whether there were significant differences among three levels and fulfilled status, such as "partly fulfilled," in Group 2. The statistical analysis was conducted with R 3.6.1 and the significance level was set as 5\%.

Since Group 1 had some outdated standards statements and testing between the levels seemed unimportant, only Group 2 was used for testing. In this study, the chi-squared test was used only to analyze the difference between areas. In addition, sub-areas included specific features of medical education and it may not reflect the general characteristics of medical education in Japan, but the characteristics of each institution. Since one of the objectives of the study was to reveal the general characteristics of medical education in Japan, analysis with sub-areas was not considered. 


\section{Results}

Group 1 had 14 institutions and Group 2 had 20 institutions. Figures 1 and 2 show the percentages of institutions that fulfilled and partly fulfilled each standard, per group.

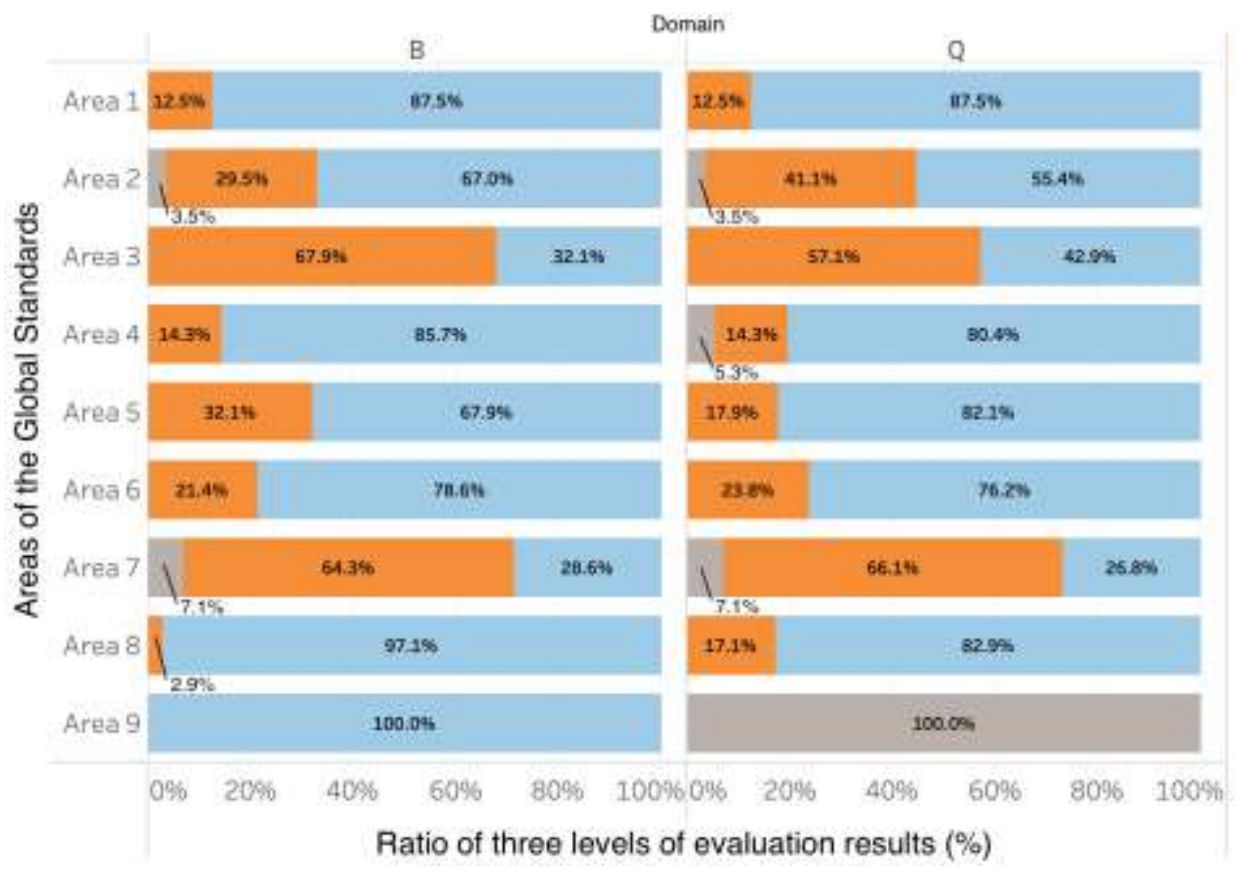

Figure 1: Group 1 (Basic and Quality Improvement)

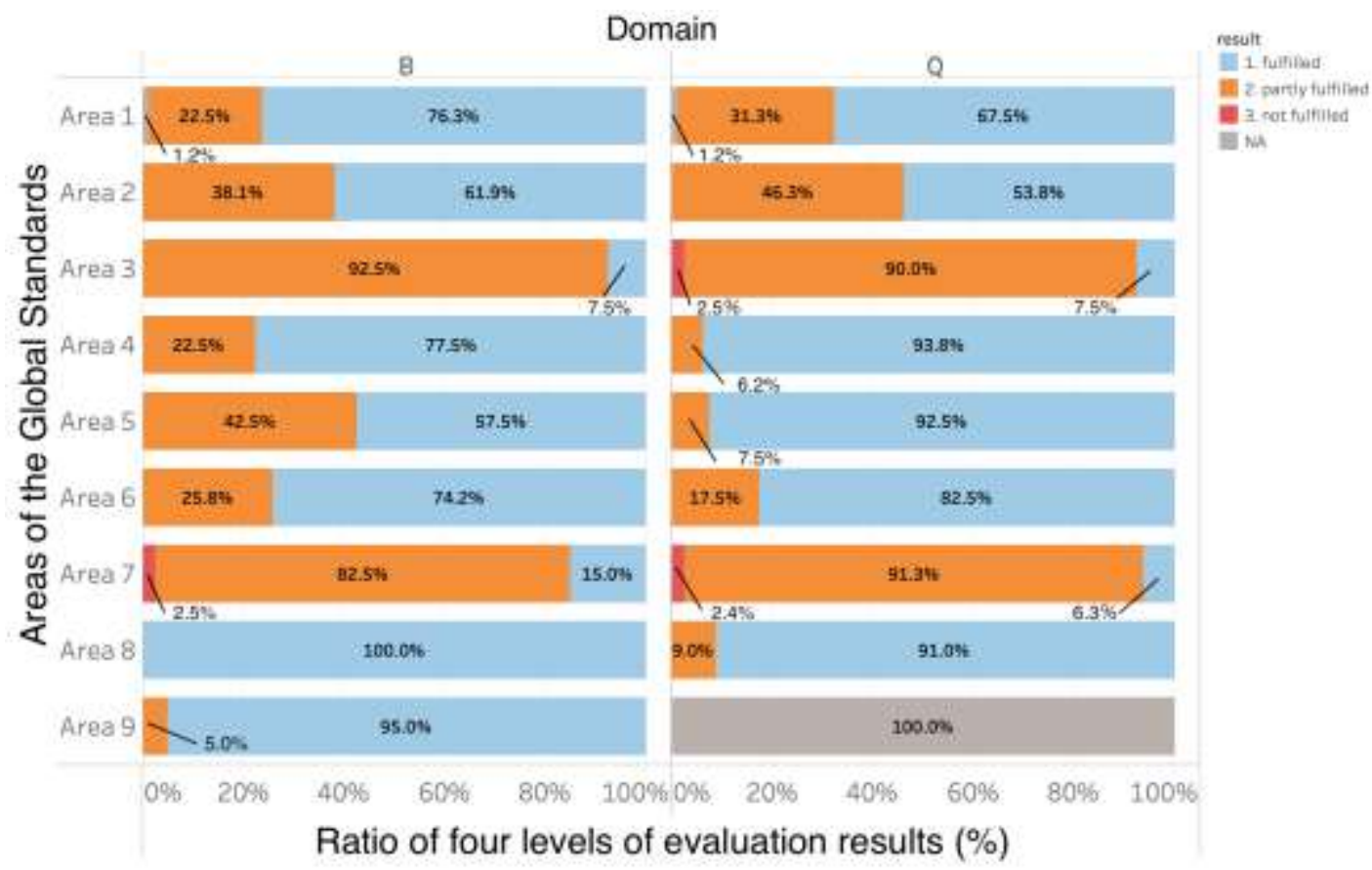

Figure 2: Group 2 (Basic and Quality Improvement) 
Although Group 1 and Group 2 used different versions of standards, these two graphs show their similar trends. For example, more than half of the institutions only partly fulfilled or did not fulfill Domain B for Areas 3 and 7. These trends were almost the same for Domain Q. Thus, additional analysis was carried out to examine the fulfillment rate of the sub-areas. There are some major revisions between 1.3 and after version 2.1, such as a number of items across the sub-areas and the materials of statements themselves. On the other hand, the differences between the minor versions are generally related to the translation and wording of Japanese sentences. For this reason, the following detailed analysis was performed only with Group 2.

It is notable that all of the elements of Domain Q for Area 9 are evaluated as "NA." Since these standards are for continuous renewal and the accreditations are the first evaluations, JACME did not evaluate these standards.

There were some other "NA" areas in both groups. In Group 1, some institutions are evaluated as "NA" in Areas 2, 4, and 7. The reasons for not evaluating these standards were included in each report, and the difficulty of defining the standards was cited as the most common reason. The sole "NA" for Group 2 pertains to the National Defense Medical College. Since this institution has a special mission and it is not appropriate to evaluate it using global standards, the evaluation was not conducted.

Table 2 shows the total number of evaluations in each sub-area of standards in Group 2. The bold numbers show which status (such as "partly fulfilled") is significantly higher or lower than other statuses. Regarding Domain B, Areas 1, 8, and 9 were significantly "fulfilled," according to the chi-squared test $\left(\chi^{2}=236.35, p<0.05\right)$. Conversely, a significantly large number of "partly fulfilled" classifications appeared in the reports for Areas 3 and 7. Regarding Domain Q, Areas 4, 5,6 , and 8 were significantly "fulfilled," whereas Areas 3 and 7 were significantly "partly fulfilled $\left(\chi^{2}=281.67, p<0.05\right)$.

Table 2: Total Count of Domain B and Domain Q Fulfilled (Group 2): significantly higher or lower statuses (such as "fulfilled") with $p<0.05$ are shown as bold numbers

\begin{tabular}{|c|c|c|c|c|c|c|c|c|}
\hline \multirow[b]{2}{*}{ Area } & \multicolumn{4}{|c|}{ Domain B } & \multicolumn{4}{|c|}{ Domain Q } \\
\hline & Fulfilled & $\begin{array}{c}\text { Partly } \\
\text { Fulfilled }\end{array}$ & $\begin{array}{c}\text { Not } \\
\text { Fulfilled }\end{array}$ & NA & Fulfilled & $\begin{array}{c}\text { Partly } \\
\text { Fulfilled }\end{array}$ & $\begin{array}{c}\text { Not } \\
\text { Fulfilled }\end{array}$ & NA \\
\hline 1 & 61 & 18 & 0 & 1 & 54 & 25 & 0 & 1 \\
\hline 2 & 99 & 61 & 0 & 0 & 86 & 74 & 0 & 0 \\
\hline 3 & 3 & 37 & 0 & 0 & 3 & 36 & 1 & 0 \\
\hline 4 & 62 & 18 & 0 & 0 & 75 & 5 & 0 & 0 \\
\hline 5 & 23 & 17 & 0 & 0 & 37 & 3 & 0 & 0 \\
\hline 6 & 89 & 31 & 0 & 0 & 99 & 21 & 0 & 0 \\
\hline 7 & 12 & 66 & 2 & 0 & 5 & 73 & 2 & 0 \\
\hline 8 & 100 & 0 & 0 & 0 & 91 & 9 & 0 & 0 \\
\hline 9 & 19 & 1 & 0 & 0 & 0 & 0 & 0 & 20 \\
\hline
\end{tabular}


The following figures show the percentage of results in each sub-area.

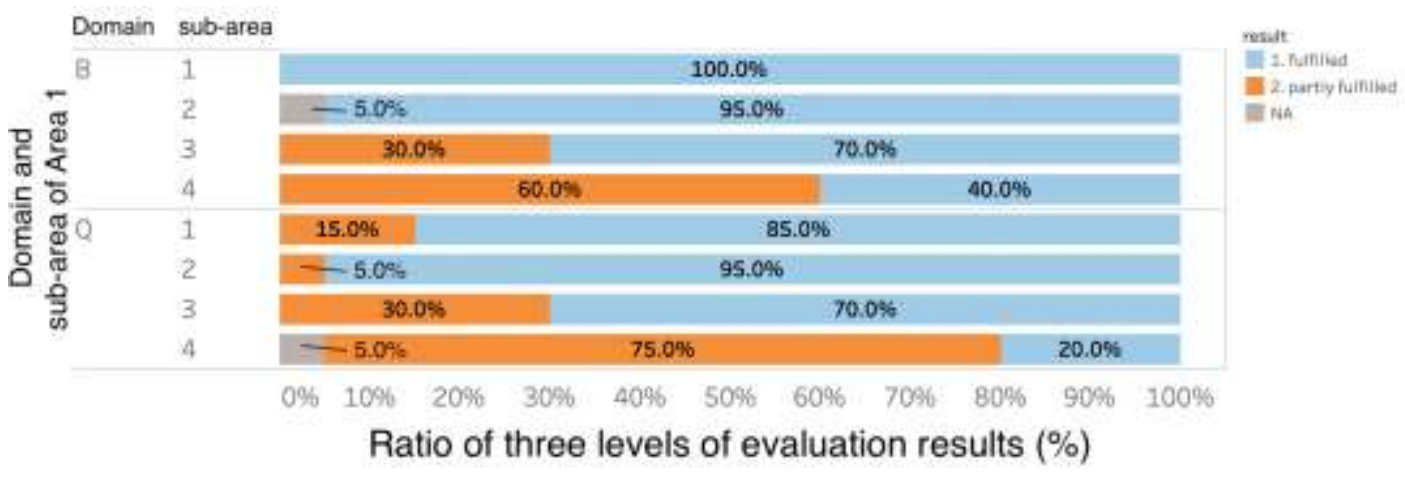

Figure 3: Sub-areas of Area 1

Figure 3 shows the results of Area 1 with four sub-areas. Some institutions were "partly fulfilled" in sub-area 1.4 both in Domain B and Domain Q. Sub-area 1.4 is the "Participation in Formulation of Mission and Outcomes." There were some comments regarding the needs of defining and aligning the stakeholders, including students and citizens, for the formulation of the mission and outcomes.

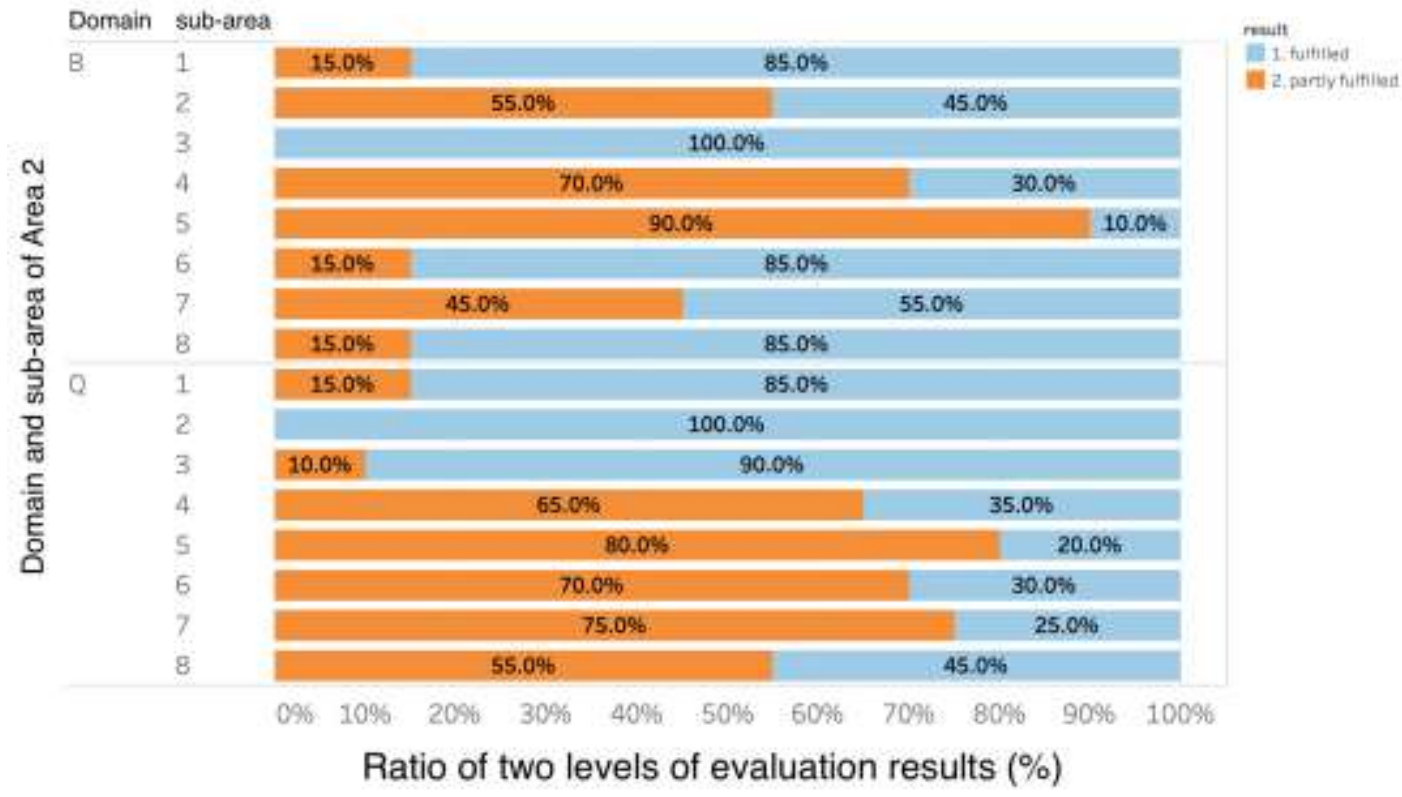

Figure 4: Sub-areas of Area 2

The results of Area 2 with eight sub-areas are shown in Figure 4. There are several "partly fulfilled" areas, such as 2.2 and 2.4. It should be noted that sub-areas 2.2, 2.4, and 2.5 are "partly fulfilled" in both domains. Sub-area 2.2 is the "Scientific Method." One of the reasons for the "partly fulfilled" classification is the lack of education about Evidence-Based Medicine (EBM). 
One of the reasons for the "partly fulfilled" classification in sub-area 2.4 is the need to define the leaders of certain subjects, such as behavior sciences. Regarding sub-area 2.5, "Clinical Sciences and Skills," there are notable comments from reports related to the need for the improvement of clinical clerkships, such as increasing participatory clerkships instead of simple observation. Sub-areas 2.6-2.8 deal with various aspects of program management. From the report, "Vertical and horizontal integration of subjects," there appears to be a need for the participation of representatives from other stakeholder groups (e.g., other health professions, patients, community) on the curriculum committee.

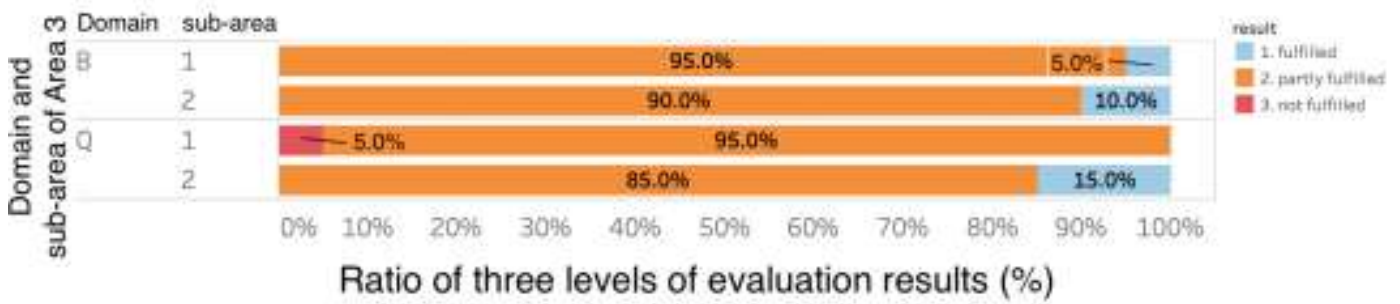

Figure 5: Sub-areas of Area 3

Figure 5 shows the results for Area 3, which has two sub-areas. There are several comments for improvements in this area. One of the most important suggestions is to build a curriculum map for competency-based education. Another notable suggestion is to decrease the number of examinations. In addition to mid-term and final exams, there are also some national exams. This examination schedule is considered to put a high level of stress on the students.

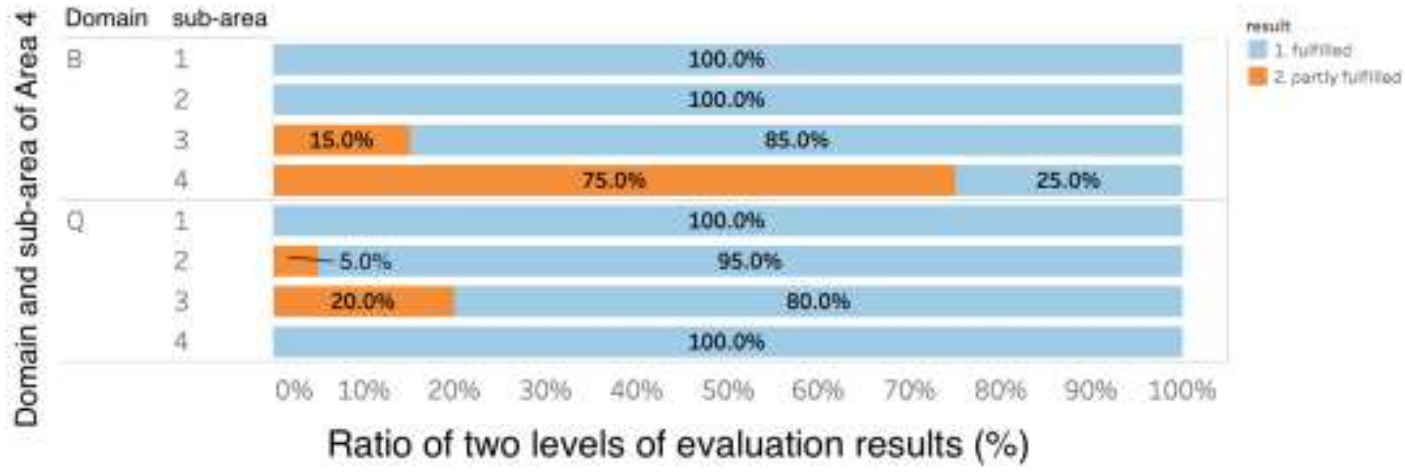

Figure 6: Sub-areas of Area 4

Figure 6 shows the results of Area 4, which includes four sub-areas. There are a lot of "partly fulfilled" institutions in sub-area 4.4 of Domain B. This sub-area is about the level of student representation present in the governance of the institutions. Fulfilling the requirements of this sub-area could prove somewhat difficult as it requires a clear policy about student representation to be reflected in the mission statement, design of the program, management of the program, and evaluation of the program. 


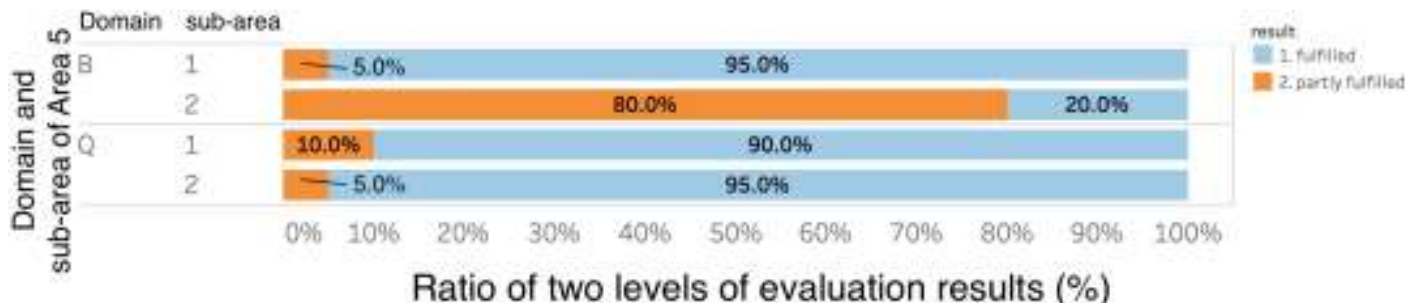

Figure 7: Sub-areas of Area 5

The results of Area 5 are shown in Figure 7 with two sub-areas. The fulfillment of sub-area 5.2, "Staff Activity and Staff Development," requires a balance of capacity between teaching, research, and service functions. Some institutions require the time spent on each of these functions to be calculated. From the reports, staff merit should be measured comprehensively, including teaching awards, professional experience, and research outputs.

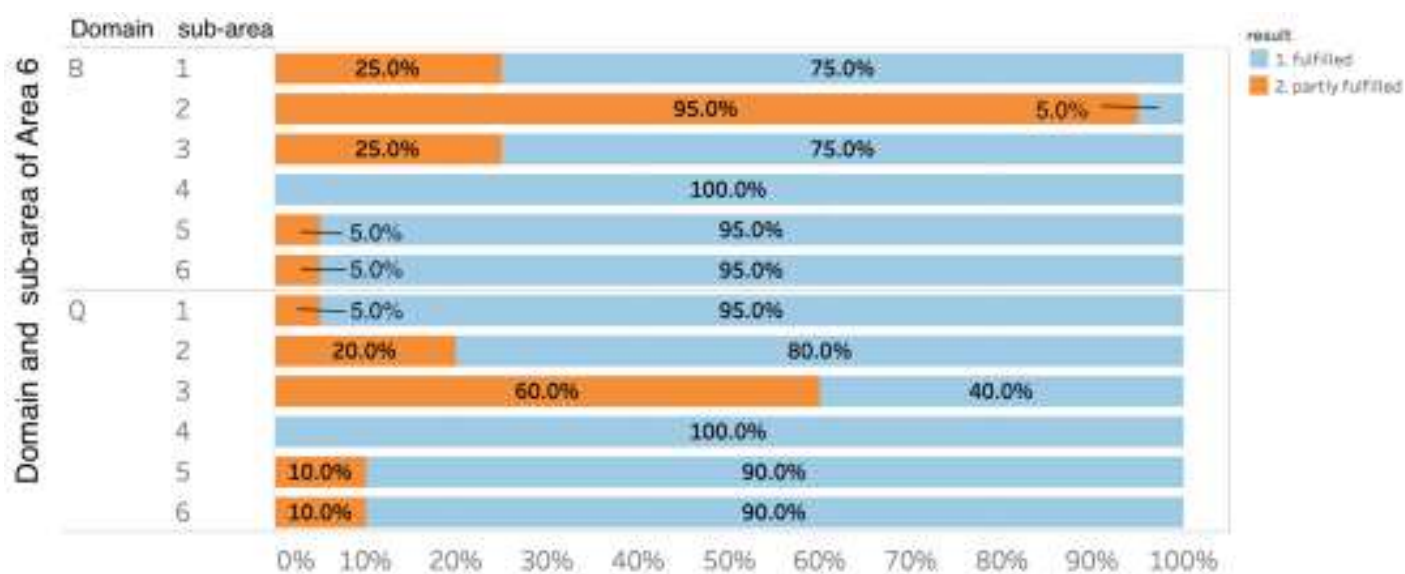

Ratio of two levels of evaluation results (\%)

Figure 8: Sub-areas of Area 6

Figure 8 shows the results for Area 6 with six sub-areas. There is notable room for improvement in sub-areas 6.2 and 6.3. Sub-area 6.2 is about the clinical training resources, and it focuses on the presence of required resources, such as the number and categories of patients, as well as clinical training facilities. Sub-area 6.3 addresses information technology for education, such as the usage of Learning Management Systems (LMS) and simulators such as manikins and virtual reality training systems. In addition, the standards for this sub-area include the maintenance of IT infrastructure, especially wireless LAN in lecture rooms and hospitals.

Figure 9 shows the results of Area 7, which has four sub-areas. This area is similar to Area 3 in that more than $50 \%$ of institutions are "partly fulfilled" across all sub-areas. In addition, some institutions were evaluated as "not fulfilled" in some sub-areas. This area is highly connected to institutional research (IR). There are comments regarding the need for "Monitoring and 
evaluation of educational programs," not only the establishment of IR itself, but the full workflow from gathering information, data management, analysis, and feedback to the stakeholders.
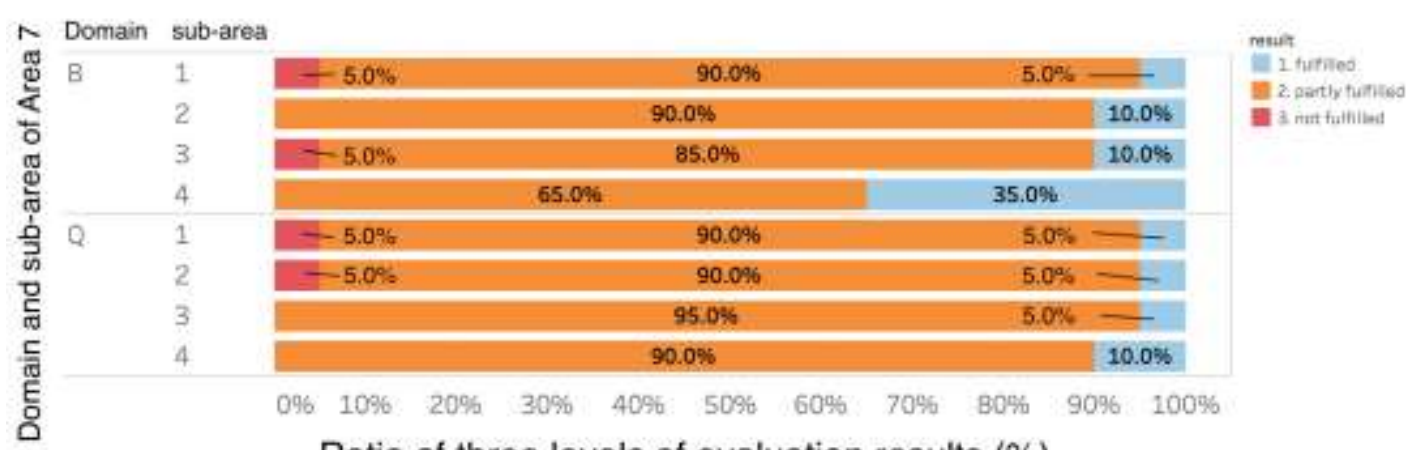

Ratio of three levels of evaluation results (\%)

Figure 9: Sub-areas of Area 7

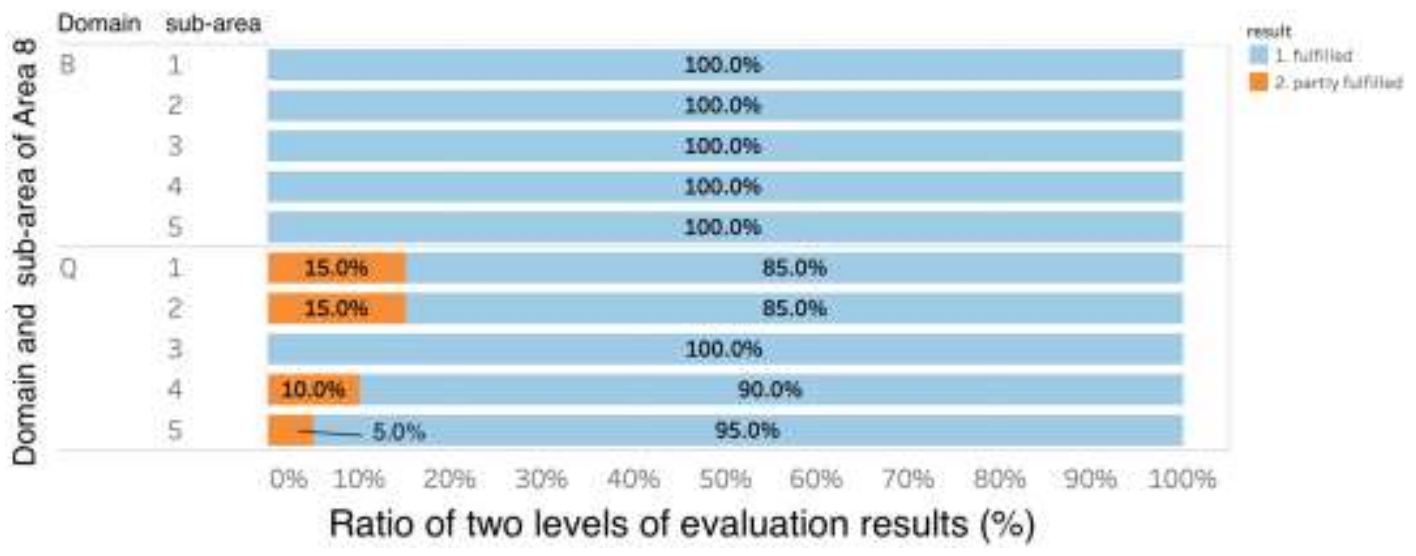

Figure 10: Sub-areas of Area 8

Figure 10 shows the results for Area 8, which includes five sub-areas. Almost all institutions are classified as "fulfilled" across both domains, with only a few exceptions in Domain Q.

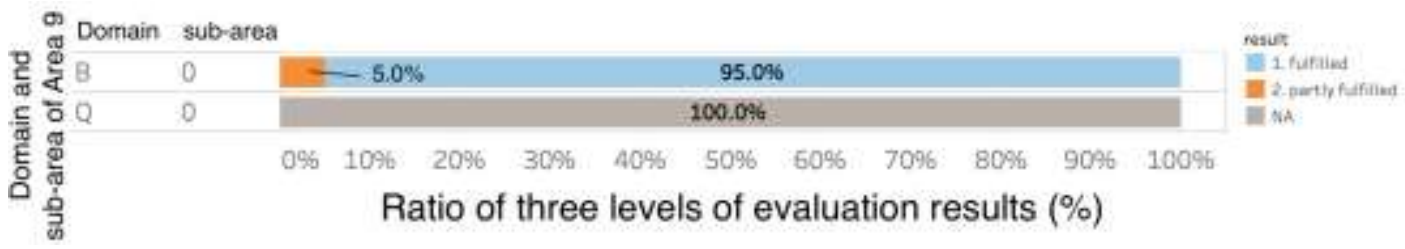

Figure 12: Sub-areas of Area 9

The results for Area 9 are shown in Figure 12. This area has only one statement. As previously noted, an evaluation was not conducted for the first accreditation in Domain Q. 


\section{Discussion}

\subsection{The current situation of Japanese medical education}

Regarding both Domains B and Q, Area 8 (Figure 10) was almost completely "fulfilled." It is possible to verify that governance and administration is one of the strongest points of medical education in Japan. Therefore, it might not be so difficult to adapt the educational system to meet global standards.

It is notable that Areas 4-6 are not significantly fulfilled regarding Domain B but are fulfilled with respect to Domain Q (Figure 6 to Figure 8). Since the elements of Domain Q are about quality improvement, they evaluate the special works of each institution. However, Domain B should be "fulfilled" before Domain Q, given their respective properties.

As mentioned above, some standards statements in Areas 1 and 4 were regarded as being unsuitable for Pakistani context [4]. Gohar et al showed that standards statements of sub-area 4-4 got significantly more negative responses than other sub-areas (Figure 6). Sub-area 4.4 is about "student representation" and three-fourths of the institutions of Group 2 were "partly fulfilled." In fact, only this sub-area was more than 50\% "partly fulfilled" in Area 4. This sub-area requires one to "formulate and implement a policy on student representation and appropriate participation," such as design and evaluation of the program. It might be difficult to make students attend the management section because of cultural or social contexts.

In both Domains B and Q, Areas 3 and 7 were "partly fulfilled" by many institutions (Figure 5 and Figure 9). They both have two sub-areas and almost the same percentage of "fulfilled" classifications. As Area 3 deals with assessments, this result shows that the assessment methods within Japanese medical education are not on par with global standards. Knowledge assessments are made constantly through methods such as computer-based testing (CBT) before clinical clerkship and the national examinations. However, skills and attitudes are not assessed as often. Although some examinations, such as the Post-Clinical Clerkship Objective Structured Clinical Examination (Post-CC OSCE), are usable for the assessments, they are not yet done in all medical institutions.

Area 3 requires the design of a curriculum map which associates each subject with the diploma policies. In Japan, there is a "model core curriculum" which is defined by the Japanese Ministry of Education, Culture, Sports, Science and Technology. This would also be the base of the curriculum mapping [3].

Area 7 focuses on program evaluation. This area is treated almost the same as IR in the accreditation process [7][8]. In fact, many institutions that were evaluated as "partly fulfilled" received comments such as "the IR section should provide educational data and feedback to the head of the Educational Affairs department and the dean."

\subsection{Suggestions for medical education in Japan based on the current situation}

As noted above, Areas 3 and 7 presented low scores compared to other areas, showing that there is some room for improvement in medical education in Japan. On the other hand, Area 8 (Figure 10) should be considered as an area of strength. Since Area 8 refers to the quality of institutional governance, it may not be very difficult to change the educational system for the fulfillment of 
global standards. In fact, some institutions use the accreditation results to advance educational reforms.

In some sub-areas, institutions are not meeting the standards of the Basic Domain but are classified as "fulfilled" in the Quality Improvements Domain. While the Quality Improvements Standards evaluate the special works of each institution and serve to strengthen Japanese medical education, the Basic Standards should be "fulfilled" first.

As shown by the results for sub-area 6.3 , there are some areas for improvement with respect to the usage of information technology, especially for Domain Q. The statements of standards of Domain B and Domain Q are as follows (from Basic Medical Education WFME Global Standards for Quality Improvement [1]):

[Domain B] The medical school must formulate and implement a policy which addresses effective and ethical use and evaluation of:

- appropriate information and communication technology

- ensuring access to web-based or other electronic media.

[Domain Q] The medical school should enable teachers and students to use existing and exploit appropriate new information and communication technology for:

- independent learning

- accessing information

- managing patients

- working in health care delivery systems

- optimal student access to relevant patient data and health care information systems.

Since the most institutions were "fulfilled" in Domain B, the basic implementation of ICT tools and learning support tools, such as LMS, has been completed. On the other hand, more than half institutions were only "partly fulfilled" in Domain Q. This means the ICT tools have just been implemented and are not yet used effectively. This was reflected in some of the feedback comments from JACME.

This might be connected to the results for Area 3. Since the results of Area 3 show only partly fulfillment of the expected standards, there is room for improvement for the assessment methods in these institutions. Although three other types of policies (admission policies, curriculum policies, and diploma policies) were defined at many institutions, the assessment policies were not equally well-defined. The lack of assessment policies might cause an ineffective usage of ICT tools, as well-established assessments and curriculum policies support their appropriate usage in areas such as independent learning.

Although institutional research is one of more important elements for the accreditation of educational programs, the expectations in the context of medical education in Japan could be considered excessive. In Japan, IR often includes other marginal areas. For example, analysis of LMS data for the prediction of at-risk students [9] is usually classified as learning analytics, but it 
is also sometimes treated as IR. Since the importance of IR is recognized in the accreditation process, and has been noted for some time within Japanese medical education, it is important to pay attention to these trends. However, this kind of misunderstanding with respect to terminology can sometimes prove inconvenient for searching the existing literature. In the preceding example, there are fewer results about "institutional research and medical education" than there are for "learning analytics and medical education."

Hans et al. suggested the use of the WFME BME Standards not only for accreditation but also for creating a quality culture requiring medical schools to be responsible for quality improvement and innovation. They also mentioned developing collegiality and understanding of the common goal of quality improvement to underpin all activities based on the standards. Although some standards might not be suitable for Japanese culture, accreditation based on global standards would be helpful to find the advantages and disadvantages of medical education and improve the medical education system in Japan.

\subsection{Limitations of the study}

As noted in section 2.1, this study used data from only 34 institutions. Although the results were based on less than $50 \%$ of the total medical universities, these universities are remarkable because they applied for the evaluation directly, rather than the universities being randomly selected for evaluation. In other words, the 34 institutions are likely to feel confident that they have good medical practices if they are requesting evaluation. This would be one reason to aggregate data from these institutions to describe the current situation and future improvements. On the other hand, the fact that only data from less than $50 \%$ of the total medical universities were included is a limitation. Therefore, more data should be gathered and investigated for a more complete study.

In this study, the author reviewed the results of the sub-areas because the reports do not include the results based on each standard. If JACME disclosed more detailed data connected to each standard, a more useful data analysis could be done. Acknowledging that it may not be appropriate to open the full level of detail, to the extent possible, JACME should summarize and publish its data with as many details as practicable in order to better reflect the current situation of Japanese medical education.

In this study, the self-evaluation reports in each institution were not used. Although the self-evaluation reports are useful to determine the advantages and disadvantages of the institution, it is difficult to analyze individual reports because each one is $200-400$ pages in length. One possible way to analyze and compare the reports is to focus on specific areas such as Area 3 and Area 7. However, it would not be suitable to investigate the current total situation of Japanese medical education. Therefore, this study used only the evaluation reports by JACME.

This study used only the results based on the number of institutions. To investigate the trends and issues in more detail, the feedback and comments from JACME should also be analyzed. 


\section{Conclusion}

In summarizing 34 accreditation reports from JACME, it was found that the level of governance and administration of medical institutions in Japan is high enough to meet global standards. However, there is still much room for improvement with respect to the quality of education itself, particularly in terms of assessments and educational resources. In addition to the need to investigate a larger sample of reports, it will also be important to find new and relevant expertise about medical education from Japan.

\section{Acknowledgement}

This work was supported by “JSPS KAKENHI” (Grant Number JP18K02865).

\section{References}

[1] Basic Medical Education WFME Global Standards for Quality Improvement, https://wfme.org/download/wfme-global-standards-for-quality-improvement-bme/?wpdmdl $=831$ (Accessed Mar. 05, 2020)

[2] Basic Medical Education WFME Global Standards for Quality Improvement with Japanese Annotations(In Japanese), https://wfme.org/download/bme-japanese-specifications-2015/?wpdmdl=834 (Accessed Mar. 05, 2020)

[3] Model Core Curriculum for Medical Education in Japan, AY 2016 Revision (in English), http://www.mext.go.jp/component/a_menu/education/detail/_icsFiles/afieldfile/2018/06/18 /1325989_30.pdf. (Accessed Mar. 05, 2020)

[4] G. Wajid, A. Sethi, R. A. Khan, H.S. A, "World Federation for Medical Education: Appropriateness of Basic Medical Education standards in Pakistan," Pakistan Journal of Medical Sciences, Vol. 35, No.5, 2019, pp. 1185-1191

[5] M. Ho, J. Abbas, D. Ahn, C. Lai, N. Nara, K. Shaw, "The "Glocalization" of Medical School Accreditation,” Academic Medicine, Vol. 92, No. 12, 2017, pp. 1715-1722.

[6] JACME (Japanese Accreditation Council for Medical Education), Results of Accreditation (in Japanese), https://www.jacme.or.jp/pdf/jacme_web_licensebanner_link.pdf. (Accessed Mar. 05, 2020)

[7] Y. Asada, "Trends of Institutional Research in Japanese Medical Education: A case study of Jichi Medical University," 2018 7th IIAI International Congress on Advanced Applied Informatics (IIAI-AAI), Yonago, Tottori, Japan, 2018, pp. 490-492.

[8] Y. Asada, "The Trend of Institutional Research in Japanese Medical Education: A Case Study from Jichi Medical University," International Journal of Institutional Research and Management, Vol. 3, No. 2, 2019, pp. 1-18. 
[9] M. Saqr, U. Fors, M. Tedre, "How Learning Analytics Can Early Predict Under-achieving Students in a Blended Medical Education Course," Medical Teacher, Vol. 39, No. 7, 2017, pp. 757-767.

[10] S. Hans, C. Leif, N. Jørgen, K. Hans, "Lessons learned from use and analysis of the WFME global standards,” Medical Teacher, Vol. 39, No. 6, 2019, pp. 650-655 